\title{
Momentum and multiplicity dependence of strangeness and nuclei production
}

\author{
Alberto Calivà ${ }^{1,2, *}$ \\ ${ }^{1}$ GSI Helmholtzzentrum für Schwerionenforschung GmbH, Planckstraße 1, 64291 Darmstadt, Germany \\ ${ }^{2}$ Physikalisches Institut, University of Heidelberg, 69120 Heidelberg, Germany
}

\begin{abstract}
Prominent phenomena observed in high-energy hadronic collisions, such as the strangeness enhancement in small collision systems with increasing multiplicity and the production of loosely bound states in collisions where extreme temperatures are reached, are still mysterious and at the center of the experimental programs of several scientific Collaborations. In this contribution, recent experimental results from the ALICE and STAR Collaborations on these two fronts are presented and discussed.
\end{abstract}

\section{Strangeness enhancement}

One of the most intriguing observations in the research field dedicated to the study of strangeness production in high-energy hadronic collisions is the continuous evolution of the ratio between (multi)strange hadron yields and pion yields with increasing charged-particle multiplicity at mid-rapidity $\left\langle\mathrm{d} N_{\mathrm{ch}} / \mathrm{d} \eta\right\rangle_{|\eta|<0.5}[1,2]$. The measured ratios show an increase for $\left\langle\mathrm{d} N_{\mathrm{ch}} / \mathrm{d} \eta\right\rangle_{|\eta|<0.5} \lesssim 50$ which is followed by a saturation for higher multiplicities, up to the most central nucleus-nucleus collisions at the LHC. This behavior seems to be independent of the collision system and the center-of-mass energy of the collision. A larger increase is observed for hadrons with larger strangeness content. The origin of strangeness enhancement is still not fully understood from the theoretical point of view and different approaches are used to describe the experimental observations. Three of the most used models are presented in the following.

In the Canonical Statistical Model (CSM) [3], the multiplicity dependence of strange hadron to pion ratios emerges from the exact conservation of electric charge, baryon number, and strangeness in the so-called correlation volume $V_{\mathrm{c}}$. In the perspective of this model, the strangeness enhancement with increasing multiplicity is rather seen as a strangeness suppression with respect to the grand-canonical limit, reached in central nucleus-nucleus collisions, going to lower multiplicities. Recent developments of this model include a multiplicitydependent chemical freeze-out temperature $T_{\text {chem }}$ and the assumption of an incomplete chemical equilibration described by a multiplicity-dependent strangeness saturation parameter $\gamma_{\mathrm{S}}$ [3]. This model provides a good description of the available data except for the $p / \pi$ ratio, which is overestimated by approximately $2 \sigma$ at all multiplicities [3]. In the two-component "core-corona" model [4], the smooth evolution from the string fragmentation regime in pp collisions to statistical hadronization in nucleus-nucleus collisions is interpreted as an increase of the relative contribution from the high-density "core" with increasing multiplicity.

\footnotetext{
*e-mail: alberto.caliva@cern.ch
} 
Strangeness enhancement can also be described in the context of the Lund string fragmentation model using high-density color strings (ropes) and the color reconnection mechanism $[5]$.

\subsection{Canonical suppression}

The role of canonical suppression becomes relevant also at low center-of-mass energy collisions. This is nicely highlighted by the measurements of $\phi / K^{-}$and $\phi / \Xi^{-}$as a function of centrality and center-of-mass energy by the STAR Collaboration (Fig. 1). The data indicate an increasing suppression of open strange hadron yields, relative to the hidden-strangeness meson $\phi$ yield, for a fixed $\sqrt{s_{\mathrm{NN}}}$ going from central to peripheral collisions. The suppression is stronger for hadrons with larger strangeness content $\left(\phi / \Xi^{-}>\phi / K^{-}\right)$. The $\sqrt{s_{\mathrm{NN}}}$ dependence of these ratios originates from an interplay between the $\sqrt{s_{\mathrm{NN}}}$ dependence of the chemical freeze-out temperature and baryochemical potential $\mu_{\mathrm{B}}$, and the effect of canonical suppression, as described by statistical hadronization models [6, 7]. Canonical suppression becomes dominant for $\sqrt{s_{\mathrm{NN}}}<4$, as indicated by the data.
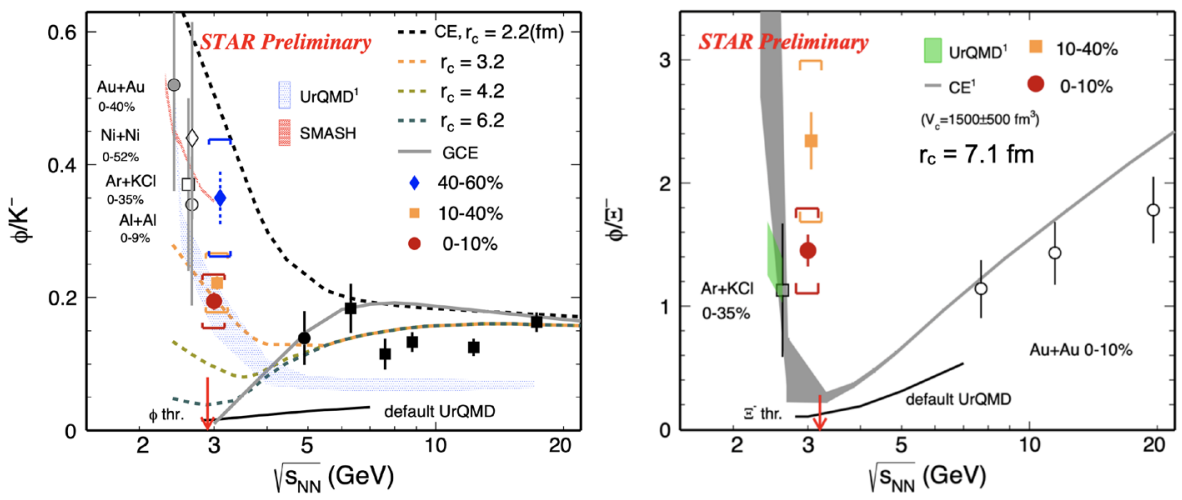

Figure 1. $\phi / K^{-}$and $\phi / \Xi^{-}$measured as a function of the center-of-mass energy in different collision systems by the STAR Collaboration.

\subsection{Strangeness production and effective energy}

Strangeness enhancement is further studied by the ALICE Collaboration as a function of effective energy. This is the energy effectively available for particle production at mid-rapidity considering that a fraction of the initial center-of-mass energy is carried away by baryons emitted at forward rapidity. Their energy is measured using the Zero-Degree Calorimeters (ZDC) and the effective energy of the collision is obtained as $E_{\mathrm{eff}}=\sqrt{s_{\mathrm{NN}}}-E_{\mathrm{ZDC}}$. Preliminary results from the ALICE Collaboration indicate that the strange hadron yields measured as a function of multiplicity in events with low and high effective energy are consistent within the uncertainties (Fig. 1). Strangeness production is therefore independent of the effective energy and is mainly driven by the final-state multiplicity.

\subsection{Strangeness production in and out of jets}

Strangeness production is further characterized by the ALICE Collaboration by measuring the yields of strange hadrons in jets and in the underlying event (UE) in pp collisions at $\sqrt{s}$ 

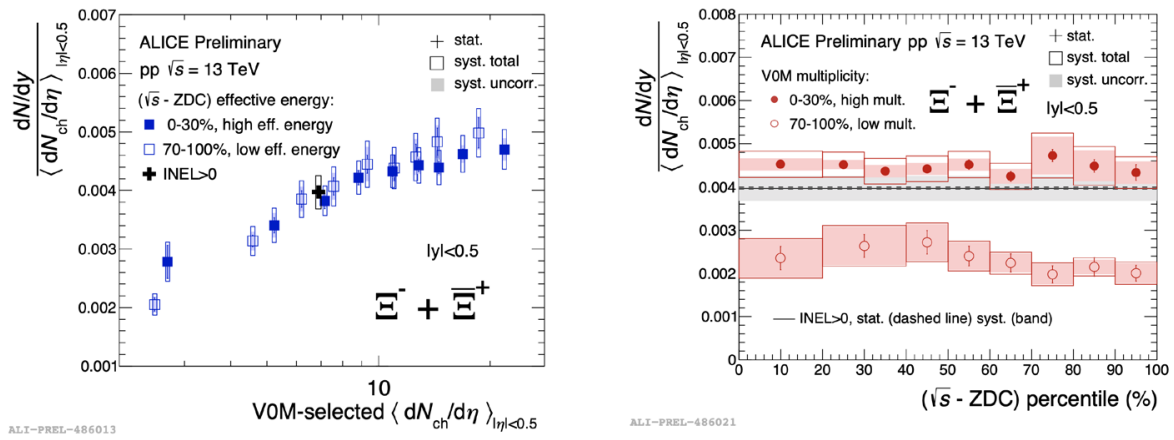

Figure 2. $\Xi$ yields, normalized by its average, shown as a function of multiplicity for low and high effective energy (left) and as a function of the $E_{\text {eff }}$ percentile for different multiplicities (right).

$=13 \mathrm{TeV}$. The two-particle correlation method is used in this measurement to distinguish the different contributions: a charged particle with the highest $p_{\mathrm{T}}$ and $p_{\mathrm{T}}>3 \mathrm{GeV} / \mathrm{c}$, called "trigger particle", is used as a proxy for the jet. The "in-jet" and "out-of-jet" regions are defined based on $\Delta \eta$ and $\Delta \phi$ of strange hadrons with respect to the trigger particle. The measured yield of strange hadrons in the UE rises faster with multiplicity than in the jet region, as shown in Fig. 3 left. The inclusive $\Xi / K_{\mathrm{S}}^{0}$ measured as a function of the event multiplicity is consistent with that measured in the UE, which implies a smaller relative contribution of strangeness production in jets. The measured ratio increases as a function of multiplicity also for the jet region with a slope similar to that in the UE. These results suggest that strangeness enhancement originates both from jets and the UE.
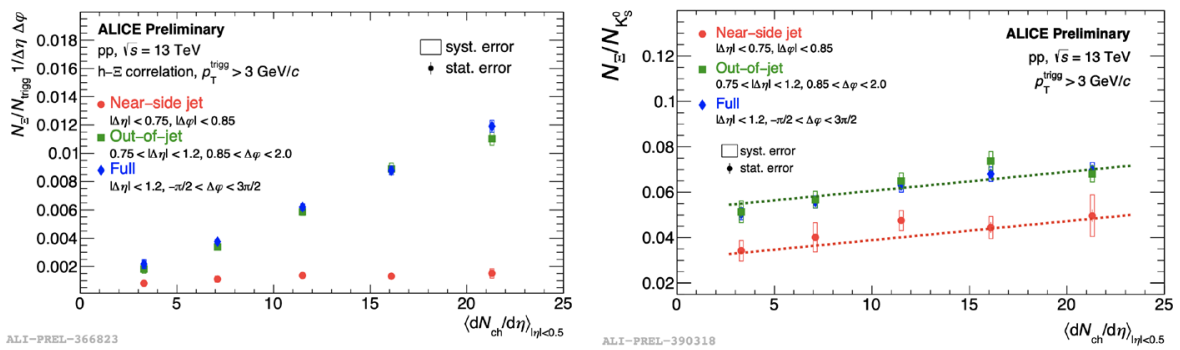

Figure 3. $\Xi$ yield density (left) and $\Xi / K_{\mathrm{S}}^{0}$ as a function of multiplicity (right) for different regions.

\section{2 (Hyper)nuclei producton}

The production mechanism of light (anti)(hyper)nuclei in high-energy hadronic collisions is still not understood and currently under intense debate in the scientific community. The experimental data are typically described using two different phenomenological models: the statistical hadronization model (SHM) and the baryon coalescence approach. In the statistical hadronization model [8-13], light (anti-)(hyper-)nuclei, as well as other hadron species, are assumed to be emitted by a source in local thermal and hadrochemical equilibrium with their abundances being fixed at chemical freeze-out, at a temperature of $T_{\text {chem }}=156 \pm 4 \mathrm{MeV}$ 
[12]. Multi-baryon states are assumed to be produced as compact multi-quark systems, which evolve towards the final configuration with typical timescales of $5 \mathrm{fm} / \mathrm{c}$ or longer [12]. This model provides an excellent description of the measured hadron yields in central nucleusnucleus collisions [12]. In the coalescence model [14-19], multi-baryon states are assumed to be formed by coalescence of baryons that are close in phase space at kinetic freeze-out. In the state-of-the-art implementations of the coalescence approach, the quantum-mechanical properties of baryons and bound states are taken into account and the coalescence probability is calculated from the overlap between the wave functions of individual baryons and the Wigner density of the final-state cluster.

From systematic studies of light (anti-)nuclei production in hadronic collisions at different multiplicities, it seems that the dominant production mechanism evolves smoothly from small to large collision systems with the charged-particle multiplicity. A smooth transition from the low-multiplicity regime towards the grand-canonical limit is observed in the yield ratios $\mathrm{d} / \mathrm{p}$ and ${ }^{3} \mathrm{He} / \mathrm{p}$. While the multiplicity evolution of the $\mathrm{d} / \mathrm{p}$ ratio is fairly well reproduced by the coalescence model [19], some tensions are observed in the intermediate multiplicity region for the ${ }^{3} \mathrm{He} / \mathrm{p}$ ratio. The canonical statistical model, with fixed $T_{\text {chem }}=155 \mathrm{MeV}$ for all multiplicities [11], provides only a qualitative description of these data. A continuous evolution with multiplicity is also observed for the coalescence parameter, defined as the ratio between the invariant yield of a nucleus with a mass number $A$ and that of protons to the power of $A$. In particular, the coalescence parameters for a fixed $p_{\mathrm{T}} / A$ continuously decrease going from pp to central nucleus-nucleus collisions. In the coalescence approach, this behavior is connected to the multiplicity evolution of the particle-emitting source size [16]: nucleons with fixed $p_{\mathrm{T}} / A$ are, on average, closer in space in small systems as compared to large systems, hence the coalescence probability in small systems is larger.

\subsection{Deuteron production in and out of jets}

The deuteron production is studied by the ALICE Collaboration in jets and in the UE in pp collisions at $\sqrt{s}=13 \mathrm{TeV}$ [20]. The $p_{\mathrm{T}}$-differential jet-associated deuteron production is measured using the two-particle correlation method as described in Sect. 1.3. The $p_{\mathrm{T}}$ spectrum of deuterons inside the jets is found to be consistent with predictions based on PYTHIA coupled to a simple coalescence afterburner. In the latter, deuteron formation is assumed to happen if a proton and neutron have a momentum difference $\Delta p<p_{0}$, with $p_{0}=110 \mathrm{MeV}$, thus ignoring the space coordinates in the calculation of the coalescence probability.

The deuteron $p_{\mathrm{T}}$ spectra are also studied in the jet region and in the UE as a function of the EU activity. The latter is quantified by the ratio $R_{\mathrm{T}}=N_{\mathrm{ch}}^{\mathrm{T}} /\left\langle N_{\mathrm{ch}}^{\mathrm{T}}\right\rangle$, where $N_{\mathrm{ch}}^{\mathrm{T}}$ is the chargedparticle multiplicity measured in the transverse region with respect to the trigger particle. Preliminary results from the ALICE Collaboration show similar coalescence parameters both in the toward and transverse regions for fixed $R_{\mathrm{T}}$. The toward region contains contributions from jets and UE, while the transverse region is dominated by the UE. The data suggest a larger $B_{2}$ in the toward region at low $R_{\mathrm{T}}$ (low UE activity), as shown in Fig. 4. PYTHIA 8.3 describes the ordering and $p_{\mathrm{T}}$ dependence of $B_{2}$ but not the magnitude.

\subsection{Hypernuclei production and their structure}

The hypertriton is a weakly bound state composed of a proton, a neutron, and a $\Lambda$. This system has a halo structure with a "deuteron" core surrounded by an extended $\Lambda$ probability cloud. The average distance between them is connected to the $\Lambda$ separation energy: the smaller $B_{\Lambda}$ the larger their distance. The widely-accepted measurement is $B_{\Lambda}=130 \pm 50$ $\mathrm{KeV}$ [21]. The hypertriton lifetime is at the center of a long-standing puzzle which consists 

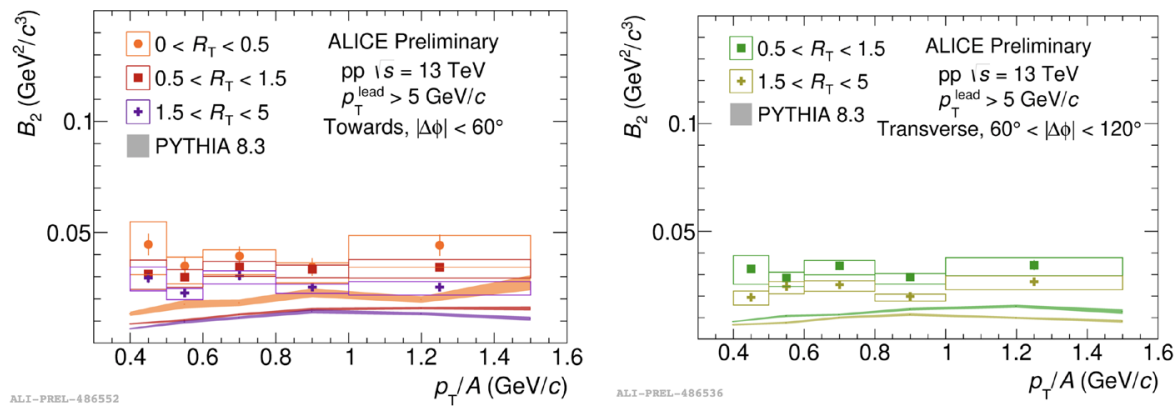

Figure 4. Coalescence parameter $B_{2}$ measured in the toward (left) and transverse (right) region for different $R_{\mathrm{T}}$ intervals.

in the difficulty to reconcile the small value of the $\Lambda$ separation energy with the relatively short lifetime measured by the experiments. In fact, most of the theory calculations, which use the $\Lambda$ separation energy as input, predict a hypertriton lifetime very close to that of the vacuum $\Lambda$, with a maximum deviation of a few $\%$. The current world average, instead, is about $30 \%$ shorter with significant tension between the published ALICE and STAR results. The most recent preliminary measurements of the hypertriton lifetime from the two Collaborations give now consistent results, which are closer to the $\Lambda$ lifetime, as shown in Fig. 5. In addition, the ALICE Collaboration has measured a $\Lambda$ separation energy consistent with zero. These new results depict a consistent picture of the hypertriton structure and the solution to the lifetime puzzle seems to be imminent.
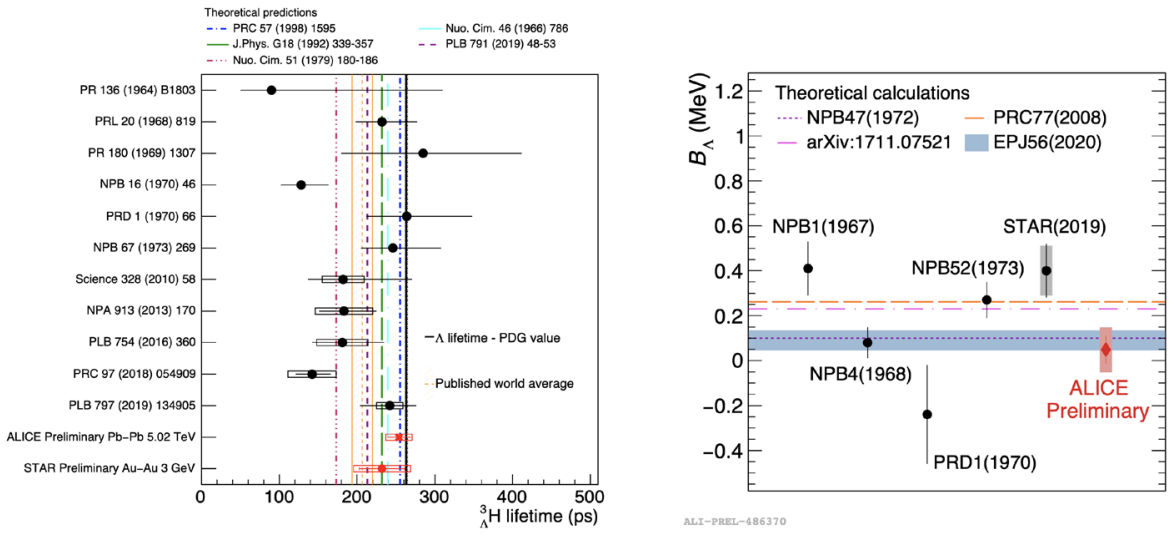

Figure 5. Hypertriton lifetime (left) and $\Lambda$ separation energy (right).

The radial extension of the hypertriton wave function, constrained by the lifetime and $\Lambda$ separation energy measurements, is a relevant parameter in the coalescence model which enters explicitly into the coalescence probability [19]. On the contrary, the predictions from the statistical hadronization model are independent of the system size and depend only on the mass and spin of the bound state [8-13]. The hypertriton yield measured in central nucleusnucleus collisions, both at low and high center-of-mass energy, are consistent with the thermal model (Fig. 6 left). On the other hand, recent measurements of the hypertriton production in 
small collision systems by the ALICE Collaboration favor the two-body coalescence model. The preliminary measurement of the ${ }_{\Lambda}^{4} \mathrm{H}$ - a bound state with two protons, a neutron and a $\Lambda$ - in central $\mathrm{Au}-\mathrm{Au}$ collisions at $3 \mathrm{GeV}$ challenges both models (Fig. 6 right).

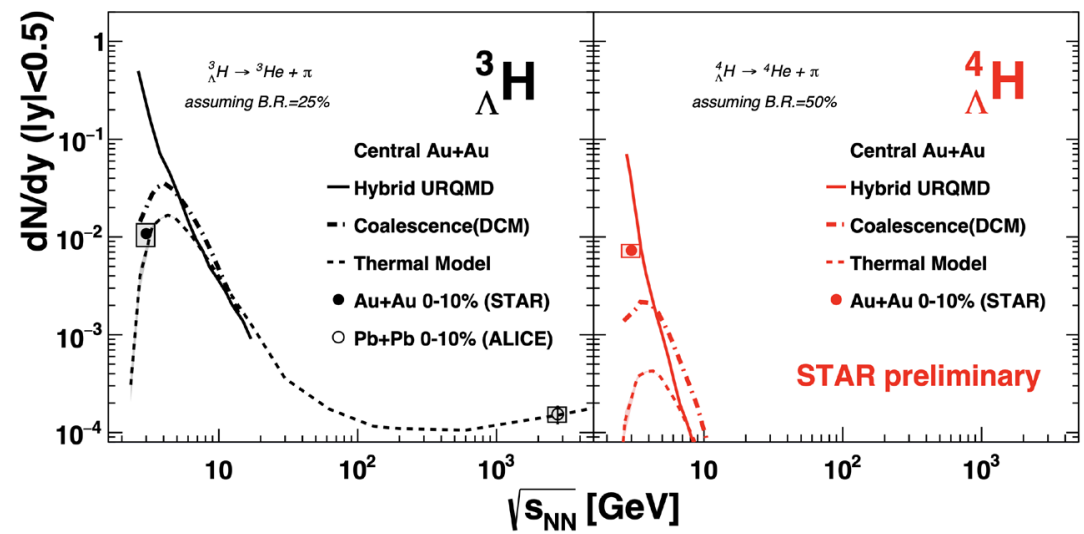

Figure 6. Hypertriton (left) and ${ }_{\Lambda}^{4} \mathrm{H}$ yield (right) as a function of the center-of-mass energy.

\section{References}

[1] J. Adam et al. (The ALICE Collaboration), Nature Phys. 13, 535-539 (2017)

[2] S. Acharya et al. (The ALICE Collaboration), Eur. Phys. J. C 80, 167 (2020)

[3] V. Vovchenko, B. Dönigus, H. Stöcker, Phys. Rev. C 100, 054906 (2019)

[4] Y. Kanakubo, Y. Tachibana, T. Hirano, Phys. Rev. C 101, 024912 (2020)

[5] R. Nayak, S. Pal, S. Dash, Phys. Rev. D 100, 074023 (2019)

[6] J. Cleymans et al., Phys. Lett. B 603, 146-151 (2004)

[7] A. Andronic, P. Braun-Munzinger, J. Stachel, Nucl. Phys. A 772, 167-199 (2006)

[8] J. Cleymans et al., Phys. Rev. C 84, 054916 (2011)

[9] A. Andronic, P. Braun-Munzinger, J. Stachel, H. Stöcker, Phys. Lett. B 697, 203-207 (2011)

[10] F. Becattini, M. Bleicher, E. Grossi, J. Steinheimer, R. Stock, Phys. Rev. C 90, 054907 (2014)

[11] V. Vovchenko and H. Stöcker, Phys. Rev. C 95, 044904 (2017)

[12] A. Andronic, P. Braun-Munzinger, K. Redlich, J. Stachel, Nature 561, 321-330 (2018)

[13] N. Sharma, J. Cleymans, B. Hippolyte, M. Paradza, Phys. Rev. C 99, 044914 (2019)

[14] S. T. Butler and C. A. Pearson, Phys. Rev. 129, 836 (1963)

[15] J. I. Kapusta, Phys. Rev. C 21, 1301-1310 (1980)

[16] R. Scheibl and U. W. Heinz, Phys. Rev. C 59, 1585-1602 (1999)

[17] W. Zhao, L. Zhu, H. Zheng, C. M. Ko, H. Song, Phys. Rev. C 98, 054905 (2018)

[18] K. Blum and M. Takimoto, Phys. Rev. C 99, 044913 (2019)

[19] K. J. Sun, C. M. Ko, B. Dönigus, Phys. Lett. B 792, 132-137 (2019)

[20] S. Acharya et al. (The ALICE Collaboration), Phys. Lett. B 819, 136440 (2021)

[21] D.H. Davis, Nucl. Phys. A 754 (2005) 